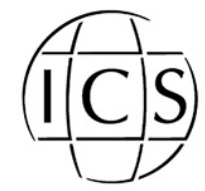

www.ics-elsevier.com

\title{
Low resolution conductivity estimation to improve source localization
}

\author{
S. Lew ${ }^{\mathrm{a}, \mathrm{b}, *}$, C. Wolters ${ }^{\mathrm{c}}$, A. Anwander ${ }^{\mathrm{d}}$, \\ S. Makeig ${ }^{\mathrm{e}}$, R. MacLeod ${ }^{\mathrm{a}, \mathrm{b}}$ \\ ${ }^{\text {a }}$ Scientific Computing and Imaging Institute, University of Utah, Salt Lake City, USA \\ ${ }^{\mathrm{b}}$ Department of Bioengineering, University of Utah, Salt Lake City, USA \\ c Institut für Biomagnetismus und Biosignalanalyse, Universität Münster, Münster, Germany \\ ${ }^{\mathrm{d}}$ Max-Planck-Institut für Kognitions- und Neurowissenschaften, Leipzig, Germany \\ e Swartz Center for Computational Neuroscience, University of California San Diego, USA
}

\begin{abstract}
Bioelectric source localization in the brain is sensitive to geometry and conductivity properties of the different head tissues. We propose a method that individually optimizes a realisticallyshaped volume conductor with regard to the conductivities using a simulated annealing optimizer in discrete parameter space. We show that the method is able to simultaneously reconstruct two reference sources and a reference skull:brain conductivity ratio even in the presence of realistic noise. The individually optimized head model can then be used for the analysis of clinical or cognitive data. (C) 2007 Elsevier B.V. All rights reserved.
\end{abstract}

Keywords: Conductivity estimation; Skull conductivity; Source localization; Simulated annealing; Optimized volume conductor

\section{Introduction}

Inverse source analysis in the brain is sensitive to the conductivities of head tissues, which vary across individuals and within the same individual due to variations in age, size, disease state, and environmental factors. One can then expect that taking such variations into consideration will improve the accuracy of source localization [1]. Here, we describe and evaluate in simulations a procedure to generate individually optimized (with regard to

\footnotetext{
* Corresponding author. Scientific Computing and Imaging Institute, University of Utah, Salt Lake City, USA. Tel.: +1 801581 7977; fax: +1 8015856513 .

E-mail address: slew@sci.utah.edu (S. Lew).
}

0531-5131/ (C) 2007 Elsevier B.V. All rights reserved. doi:10.1016/j.ics.2006.12.058 


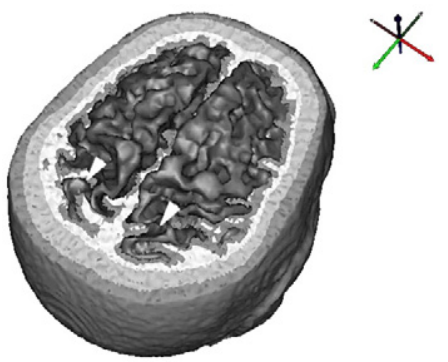

Fig. 1. Realistic four compartment (scalp, skull, CSF, brain) finite element head model with two tangentially oriented somatosensory dipoles (white arrows) on the influence space (innermost layer).

both geometry and conductivity) realistic head models, based on individual MRI and/or CT image sets and EEG data from simple evoked potentials (e.g., SEP, AEP) studies.

\section{Methods}

We created a four compartment (skin, skull, CSF, brain) tetrahedral finite element volume conductor model with a resolution of $1.5 \mathrm{~mm}$ ( $245 \mathrm{~K}$ nodes, $1503 \mathrm{~K}$ elements) from MR images of a human head (Fig. 1). For the reference volume conductor, we used conductivity values of $0.33,0.0132,1.79$ and $0.33 \mathrm{~S} / \mathrm{m}$ for the skin, skull, CSF, and brain compartments, respectively, i.e., a skull:brain ratio of 1:25. The discrete influence space consisted of a $2 \mathrm{~mm}$ triangular mesh $(21 \mathrm{~K}$ influence nodes, $43 \mathrm{~K}$ elements) located $2 \mathrm{~mm}$ beneath the cortical surface (Fig. 1). Reference sources were tangentially oriented dipoles on influence nodes in the right and left somatosensory cortex (Fig. 1).

Seventy one electrodes were equally spaced over the scalp at which sites we computed EEG reference potentials for the two simultaneously activated somatosensory sources. We then added white Gaussian noise to a SNR of 40, 25 and 20dB (Fig. 2). We used an FE direct potential approach (principle of Saint Venant, see [2,3]) with linear basis functions and solved the resulting system of linear equations by means of an Algebraic MultiGrid Preconditioned Conjugate Gradient (AMG-CG) method (relative accuracy of $10^{-8}$ ) [4].

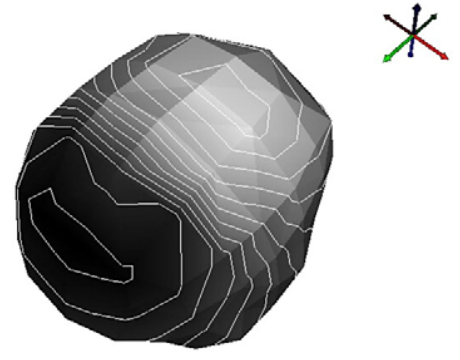

Fig. 2. Reference EEG potentials $(25 \mathrm{~dB})$ for the simultaneously activated somatosensory sources linearly interpolated on the electrode cap (isopotentials as white lines, black indicates positive potentials and white negative potentials) with a skull:brain conductivity ratio of 1:25. 

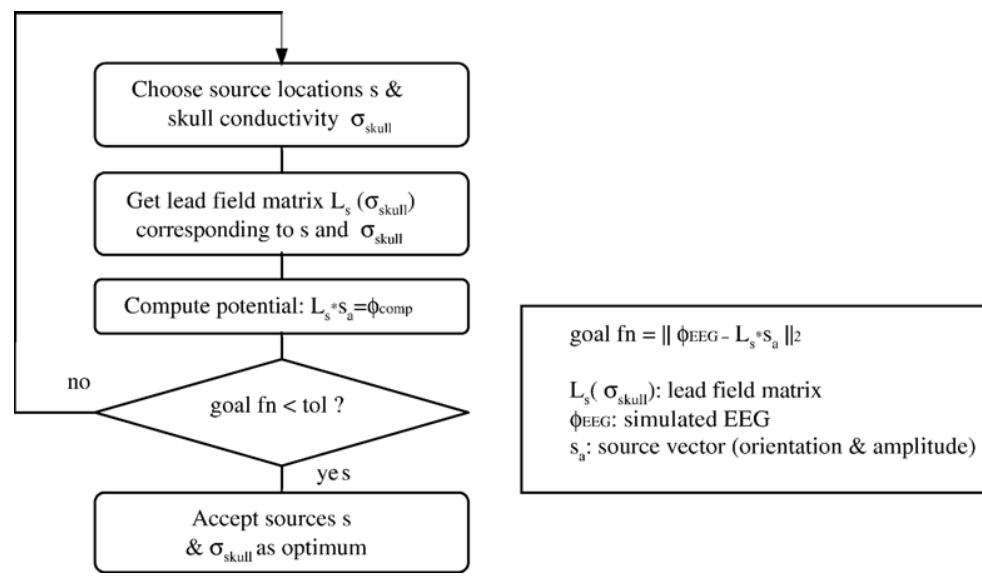

Fig. 3. Procedure of conductivity fitting with simulated annealing optimizer.

Using the simulated noisy reference EEG data and FE volume conductors with the fixed reference conductivities for the scalp, CSF, and brain compartments, we performed an inverse 2-dipole fit on the discrete influence space, while additionally allowing the skull conductivity $\left(\sigma_{\text {skull }}\right)$ to vary as a free discrete optimization parameter among the skull:brain ratios of 1:80, 1:40, 1:25, 1:15, 1:10, 1:8, and 1:5.

While exploiting the fast lead field basis approach described in [4], we precomputed lead field matrices for all conductivity ratios for the given electrodes and the influence space and used the result as an input to the following optimization procedure.

Conductivity estimation used a Simulated Annealing (SA) optimizer [5] with a temperature factor of 0.97 to search for an optimal configuration consisting of both source locations and a skull conductivity that minimize the goal function defined as the L2 norm of the difference between the reference EEG and the computed potentials (Fig. 3).

\section{Results}

The localization errors, i.e., the distance between the reference somatosensory source locations and the inversely fitted dipole locations on the influence space, and the fitted skull to brain conductivity ratio are shown for different noise levels in Table 1 . The optimized conductivity ratios for all noise levels corresponded to the reference ratio of 1:25.

Table 1

Localization error and fitted skull conductivity for conductivity fitting

\begin{tabular}{llll}
\hline EEG data & Dipole \#1 $(\mathrm{mm})$ & Dipole $\# 2(\mathrm{~mm})$ & Fitted skull:brain conductivity ratio \\
\hline No noise & 0.000 & 0.000 & $1: 25$ \\
$40 \mathrm{~dB}$ noise & 0.000 & 2.236 & $1: 25$ \\
$25 \mathrm{~dB}$ noise & 3.329 & 2.008 & $1: 25$ \\
$20 \mathrm{~dB}$ noise & 5.941 & 6.025 & $1: 25$ \\
\hline
\end{tabular}


Table 2

Localization error for fixed skull conductivity

\begin{tabular}{lcc}
\hline Fixed skull:brain conductivity ratio & Dipole \#1 $(\mathrm{mm})$ & Dipole \#2 $(\mathrm{mm})$ \\
\hline $1: 80$ & 10.816 & 12.702 \\
$1: 40$ & 11.228 & 3.757 \\
$1: 25$ & 3.329 & 2.008 \\
$1: 15$ & 10.725 & 3.175 \\
$1: 10$ & 10.722 & 2.246 \\
$1: 8$ & 10.722 & 7.101 \\
$1: 5$ & 20.531 & 3.330 \\
\hline
\end{tabular}

Table 2 shows the localization error of the SA 2-dipole fit for a range of skull:brain conductivity ratios without the conductivity fitting. The simulated EEG used for the source localization had $25 \mathrm{~dB}$ SNR.

\section{Discussion}

We evaluated a conductivity estimation algorithm using EEG simulations to determine the skull:brain conductivity ratio. Our results suggest that this procedure is able to improve source localization because it assigns accurate material properties in customized volume conductor models. The success of our conductivity optimization approach and the more general advantages of customized geometric models suggest a procedure for clinical applications. First of all, one could use evoked somatosensory signals (SEP/SEF) together with MR images from the patient to construct a model that would be optimized for both geometric accuracy and realistic conductivity values. With this model in place, recorded potentials from more complex and clinically interesting sources could drive the inverse solution and source localization.

\section{Acknowledgements}

This research was supported by the Center for Integrative Biomedical Computing, NIH NCRR Project 2-P41-RR12553-07 and by the German Research Foundation (DFG Project JU445/5-1).

\section{References}

[1] D. Gutierrez, A. Nehorai, C.H. Muravchik, Estimating brain conductivities and dipole source signals with EEG arrays, IEEE Trans. Biomed. Eng. 51 (2004) 2113-2122.

[2] C.H. Wolters, et al., Numerical approaches for dipole modeling in finite element method based source analysis, International Congress Series 1300, 15th Int. Conf. on Biomagnetism, Vancouver, BC Canada, Aug. 20-26, in review.

[3] H. Buchner, et al., Inverse localization of electric dipole current sources in finite element models of the human head, Electroencephalogr. Clin. Neurophysiol. 102 (1997) 267-278.

[4] C.H. Wolters, L. Grasedyck, W. Hackbusch, Efficient computation of lead field bases and influence matrix for the FEM-based EEG and MEG inverse problem, Inverse Problems 40 (2004) 1099-1116.

[5] S. Kirkpatrick, C.D. Gelatt Jr., M.P. Vecchi, Optimization by simulated annealing, Science 220 (1983) 671-680. 